\title{
Los desafíos en una pedagogía de cultura de paz crisis de la escuela, crisis del adulto'
}

\section{Resumen}

Anastasio Lovo nos entrega una reflexión sobre seis problemas contemporáneos capitales en el proceso enseñanza-aprendizaje de la educación en todas sus áreas. El autor adapta a la realidad de un país dependiente y empobrecido como lo son Nicaragua y gran parte de los países de América Latina, el aporte de dos pedagogos italianos Luigi Tufanelli y Dario lanes, quienes en su obra "La gestión de la clase", descubren estos desafíos globales para la educación. Este artículo es una contribución a la reflexión sobre aquellos problemas que están lesionando el proceso, donde educandos y educadores, construyen la formación técnica y profesional en el mundo. Un texto de imprescindible lectura para el profesorado que gestiona clases en cualquier nivel académico.

Palabras clave: Pedagogía, enseñanza-aprendizaje, desafíos.

\section{A los abnegados maestros de Nicaragua. Al cuerpo docente de la UPOLI. A los profesores y promotores de Cultura de Paz.}

\section{.}

- Anastasio Lovo gives us a reflection on six contemporary capital : problems in the teaching-learning process of education in all - its areas. The author adapts to the reality of a dependent and - impoverished country such as Nicaragua and most of the countries - of Latin America, the contribution of two Italian pedagogues Luigi - Tufanelli and Dario lanes, who in their work "The management - of the class", discover these global challenges for education. - This article is a contribution to the reflection on those problems - that are damaging the process, where students and educators, - build the technical and professional formation in the world. An - essential reading text for teachers who manage classes at any - academic level.

: Key words: Pedagogy, teaching-learning, challenges.

Hay una fatiga de enseñar a causa de:

1. Primera hipótesis: la pérdida del prestigio social del enseñante;

2. segunda hipótesis: un modelo de enseñanza que no funciona más;

3. tercera hipótesis: una base cultural pobre;

4. cuarta hipótesis: la televisión una mala maestra;

5. quinta hipótesis: el foso digital; y;

6. sexta hipótesis: la crisis del adulto. por Tuffanelli e Ianes y que considero comunes en la crisis de la escuela universal contemporánea, son los siguientes:

1 Este artículo es un fragmento de una investigación documental mayor publicada con el título "Pedagogía de la Paz" en el volumen colectivo La rama de olivo. Una cultura de paz global, Hispamer-IMLK UPOLI, Managua, 2016.

2 Poeta y escritor nicaragüense. Presidente Honorario del Centro Nicaragüense de Escritores (CNE). 


\section{La pérdida del prestigio social del maestro/a}

En países capitalistas dependientes como Nicaragua, la "fatiga de enseñar" se hace sentir más severamente dada las limitaciones de todo tipo: físicas y ambientales, tecnológicas, de actualización en conocimientos y métodos pedagógicos; y aún más, decisivamente, a esta fatiga contribuye angustiosamente el aspecto salarial. De toda Centroamérica, para no ir muy lejos, las maestras y los maestros nicaragüenses son los que padecen los peores salarios.

El eminente pedagogo nicaragüense, Carlos Tünnermann Bernheim, escribió sobre el particular en el artículo de opinión "El salario de los maestros", publicado en El Nuevo Diario el 05 de mayo de 2010, los siguientes párrafos cuyos contenidos 7 años después están vigente:

Los maestros, justificadamente, demandan un incremento de salario. En Nicaragua, a diferencia de otros países, el magisterio es uno de los sectores peor pagados si se toma en cuenta el esfuerzo que significa el trabajo de aula. El salario básico promedio de un maestro de Primaria anda por los tres mil quinientos a cuatro mil córdobas y el de un profesor de Secundaria ronda los cuatro mil quinientos córdobas. Son salarios notoriamente insuficientes, si consideramos que el costo de la canasta básica de 53 productos se estima en casi nueve mil córdobas, lo que significa que con su salario un maestro o profesor sólo puede adquirir menos de la mitad de esos productos. (...)

La demanda de un salario decente para el magisterio es una asignatura pendiente en la agenda nacional. No sólo se trata de un justo reclamo laboral sino que tiene que ver con el buen desempeño del Sistema Educativo Nacional. Estudios realizados por organismos internacionales señalan que "la calidad de un sistema educativo tiene como techo la calidad de los docentes". Y la calidad de los docentes tiene que ver no sólo con su formación sino también con el atractivo de la carrera docente, los salarios, los estímulos, las prestaciones sociales y el status que la sociedad les confiere a los maestros.
El "Plan Nacional de Educación 2001-2015", que no ha sido sustituido por otro, contempla: "El incremento del salario básico para maestros y maestras de educación no superior en dos momentos: de 2001 a 2005 alcanzar progresivamente el equivalente al costo de la canasta básica oficial; y de 2006 a 2010 alcanzar el salario promedio centroamericano" (página 37 del Plan) que equivale, aproximadamente, a US\$ 400 dólares. Han transcurrido varios años desde que se aprobó el "Plan Nacional de Educación 2001-2015", y los sucesivos gobiernos no han hecho los esfuerzos necesarios para que se cumplan las metas del mismo, ni siquiera de manera gradual. Al contrario, pareciera que nos alejamos de ellas cada vez más.

La pobre y dura realidad salarial y la baja estima y desprecio del status social concomitante al salario del educador y la educadora nicaragüense, tiene como resultados que cada día sean menos los interesados en esta noble profesión en nuestro país. Definitivamente asistimos a una mengua del prestigio social del educador que nunca en nuestro país ha sido justipreciada.

Sobre este particular es muy significativa y esclarecedora la Nota de El Nuevo Diario del 29 de junio 2013 Día del Maestro Nicaragüense, publicada bajo el título de "El dolor de ser maestro", cito:

El catedrático Rafael Lucio Gil, Coordinador Académico del Instituto de Educación de la Universidad Centroamericana, IDEUCA, aseveró que la mejor manera de incentivar al magisterio nacional es pagando un salario digno. "Se debe valorar la formación docente, crear políticas de apoyo al maestro como e 1 reconocimiento social, aumento salarial mejores condiciones de trabajo, ya que esto elevará la calidad en la educación del país", refirió Lucio Gil. Indicó que esto se logrará asignando un mayor presupuesto a la educación, ya que el actual corresponde al 3.5\% del Producto Interno Bruto, y lo

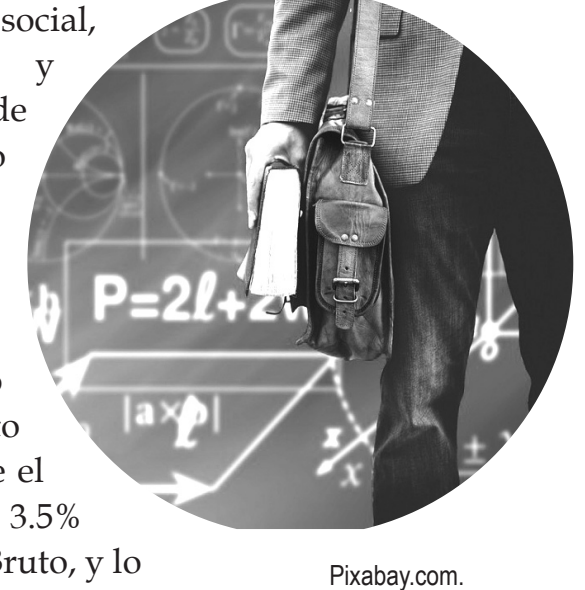

Pixabay.com. 
ideal sería llegar al 7\% del PIB, tomando en cuenta que son más de 40 mil maestros en todo el país. " $\mathrm{La}$ Ley General de Educación establece que año con año se aumente el salario del docente, pero no precisa de cuánto será ese incremento. Debido al Fondo Monetario Internacional, FMI, la ley quedó inodora, incolora e insípida, ya que no establece un monto preciso", dijo el coordinador del IDEUCA.

Mientras tanto, todos los gobernantes que han pasado aseguran que el maestro merece un mejor salario por su apostolado, pero eso no lo reflejan en sus salarios.

\section{Un modelo de enseñanza que no funciona más}

Sobre la segunda hipótesis referida a un modelo de enseñanza que no funciona más, no vamos a citar las posturas radicales sobre la muerte de la escuela de Bruce Reimer, la sociedad desescolarizada de Ivan Ilich, la deseducación obligatoria de Paul Goodman, la escuela como reproductora de la violencia simbólica de Pierre Bordieu, o la escuela en casa y crecer sin escuela de John Holt.

Más bien nos referiremos aquí a las deplorables condiciones ambientales en el que se desarrolla el proceso enseñanza-aprendizaje en la mayoría de las escuelas de los países dependientes. Como dice en un artículo sobre las "Condiciones ambientales en la escuela", la Profesora Martha Vásquez Reina:

Los espacios escolares influyen sobre los resultados académicos de los alumnos. Así lo confirman distintas investigaciones realizadas en torno al impacto que las condiciones ambientales y ergonómicas de los centros educativos tienen en el rendimiento de los estudiantes y en la labor de los docentes.

El profesor de la Universidad de Durham (Reino Unido) Steve Higgins, en su revisión literaria 'El impacto de los ambientes educativos', subraya una evidencia clara de que "ambientes de aprendizaje extremadamente pobres tienen un efecto negativo en los estudiantes y el personal docente", así como que, al mejorarlo, "se obtienen beneficios significativos".
La calidad interna del aire, ventilación y confort térmico, iluminación, acústica y el tamaño de la escuela y de las aulas son algunos de los atributos espaciales que pueden incidir sobre el rendimiento de alumnos y profesores, tal como apunta Mark Schneider, del Centro Nacional de Estadísticas Educativas estadounidense. Su trabajo de investigación ¿Afectan los espacios educativos a los resultados académicos?, concluye que "son necesarios aire limpio, buena iluminación, y un ambiente de aprendizaje tranquilo, cómodo y seguro".

\section{Una formación cultural pobre}

Sobre la tercera hipótesis de Tuffanelli y Ianes referida a una formación cultural pobre, podemos señalar que este problema se extrema en las condiciones propias de los países dependientes donde el foso entre "cultos" e iletrados es mayor y donde la segregación de seres marginales es aún más aguda que en los países metropolitanos o centrales.

La formación cultural pobre, provoca un bajo desarrollo científico técnico que redundará específicamente en los índices de desarrollo humano de los países y por supuesto impactará en primer lugar, en la esfera del sistema de educación haciéndola desagradable, aburrida, lenta, incompleta y no pertinente el funcionamiento del mismo. Para los países dependientes que padecen un foso, que no era una brecha científica y técnica con los países desarrollados, esta situación ha devenido en un círculo vicioso que alcanza únicamente a ahondar más en la marginalidad y en la prescindibilidad de estas sociedades dependientes. Así de dramática y peor es la situación real.

Según datos del Instituto de Estadística de la UNESCO, 793 millones de adultos son analfabetos, en su mayoría chicas y mujeres. Otros 67 millones de niños en edad de asistir a la escuela primaria no lo hacen y 72 millones de adolescentes en edad de cursar el primer ciclo de la enseñanza secundaria tampoco están gozando de su derecho a la educación.

Once países del mundo tienen más de $50 \%$ de adultos analfabetos. Se trata de Benin, Burkina Faso, Chad, Etiopía, Gambia, Guinea, Haití, Mali, Níger, Senegal y Sierra 
Leona. Por regiones, el sur y el oeste de Asia albergan más de la mitad de la población analfabeta mundial $(51,8 \%)$, en tanto que en el África Subsahariana viven el 21,4\% de los adultos analfabetos, en Asia Oriental y el Pacífico el 12,8\%, en los Estados Árabes el 7,6\% y en América Latina y el Caribe el 4,6\%. América del Norte, Europa y Asia Central suman por su parte el $2 \%$ de los adultos analfabetos. (UNESCOPRESS, 0509-2011).

Por otro lado:

Las estadísticas del Banco Mundial son más benévolas con América Latina, donde persisten situaciones graves de exclusión, si bien se han conseguido avances significativos en la reducción de las tasas de analfabetismo. (...) En América Latina la desigualdad es la nota dominante. La mayor tasa de personas adultas analfabetas se registra en Guatemala, Nicaragua, Honduras y El Salvador, mientras que Argentina, Chile y Uruguay cuentan con las tasas más bajas. En medio se sitúan el resto de países. Las áreas rurales albergan un mayor número de personas analfabetas, en contraposición a lo que sucede en las ciudades, por lo que salir de la situación de pobreza es aún más difícil para quienes no residen en áreas urbanas. (Azucena García 2012: "Analfabetismo: todavía uno de los males que afectan a la población mundial, Eroski Consumer", Internet).

\section{La televisión: una mala maestra}

En relación a la cuarta hipótesis de Tuffanelli e Ianes, referida a la televisión como una mala maestra, ellos señalan que es inevitable preguntarse: ¿Qué impacto pueden tener las nuevas y permisivas tecnologías de la información y la comunicación sobre una población adulta que presenta un bajo nivel de alfabetización funcional en el caso de los padres de familia? ¿Y de reflejo, que incidencia puede tener sobre la educación de los hijos?

La televisión sigue siendo el medio electrónico más amado por los niños en los países dependientes y en los países desarrollados. Veamos algunos datos tomados al azar de Internet:

En promedio, los niños ven más de 4 horas de televisión al día en Estados Unidos. Esto a pesar de que la Academia Estadounidense de Pediatría (AAP por sus siglas en inglés) recomienda que los pequeños mayores de 2 años, vean no más de una a dos horas de televisión diariamente. La AAP además sugiere evitar que los niños menores de 2 años vean la tele." http://espanol. babycenter.com/a2600196/los-ni\%C3\%B1os-y-latelevisi\%C3\%B3n\#ixzz2hgNubYwR.

En México, un niño ve en promedio 2 mil horas de televisión abierta, mientras que a la escuela sólo le dedica menos de 800 horas en un año escolar. De este modo, este medio se convierte en la "niñera electrónica", cuya influencia se da en dos niveles: salud y nutrición, y transmisión de valores y formas de interacción social.

De acuerdo con el Instituto Nacional de Estadística, Geografía e Informática, (INEGI), los menores mexicanos ven de dos a tres horas diarias de televisión, tiempo que suele triplicarse durante las vacaciones.

Ante este hecho, Wulfrano Torres Pérez, doctor en Psicología Social e investigador de la Benemérita Universidad Autónoma de Puebla, consideró que la televisión lejos de educar, des-educa, en tanto que "transmite valores y formas de interactuar que no tienen nada de humano, como son la estupidez, la violencia y la exaltación de lo tonto y superfluo. (Los niños mejicanos secuestrados por la televisión por el Dr. Wulfrano Torres Pérez en http://www. statuspuebla.com.mx/index.php?option=com_cont ent\&view $=$ article $\& i d=2013 \&$ catid $=52$ :buapenstatus \&Itemid=58).

"Cuidado la televisión puede frenar el desarrollo de la niñez menor de tres años, causar retardo psicomotor, fortalecer la pasividad, causar sobre excitación y disturbios del sueño." Esta advertencia la hacen la Autoridad para la Comunicación y el Ministerio de Salud de Francia al inicio de un programa infantil producido y enlatado en el Reino Unido.

Sin situarse en la perspectiva de los apocalípticos (Apocalípticos e integrados, Umberto Eco, 1968), la televisión comercial que padecen países dependientes como el nuestro es la peor de las maestras. La forma descarnada de presentar 
la nota roja televisiva y radial ha llegado en Nicaragua, a extremos de insania y crueldad sencillamente inadmisible y por consecuencia deformadoras de la condición humana; y en la práctica de hecho la nota roja mediática actúa como verdadero terrorista en contra de la pedagogía de la paz.

En Nicaragua el eminente filósofo humanista y pedagogo vasco-nicaragüense Juan Bautista Arríen, en su artículo "Introducción a la violencia en la educación de Nicaragua en el marco del estudio "Historia y Violencia en Nicaragua, deja consignada a la televisión entre los factores propiciadores de la violencia en nuestro país: "Los medios de comunicación en particular la TV en cuyos espacios se exhiben diferentes escenas de violencia." (Arríen 2012)

Leamos lo que dice el especialista en comunicación social, Guillermo Rothschuh Villanueva en su libro Puntos sobre las ies (Cinco, Managua, 2012), acerca de la violencia proyectada desde los noticieros Acción 10 de Canal 10 y Crónica de Canal 8:

La nota roja es eminentemente discriminatoria. Las mujeres pobres son quienes aparecen en las pantallas de Crónica y Acción 10. La persecución del rating no debe ser la única norma que sirva de presupuesto a los canales de televisión. En su afán por obtener el primer lugar pierden de perspectiva valores humanos trascendentales. En vez de propiciar la paz familiar, la nota roja atiza el fuego. Elimina el sentido de compasión y solidaridad. Las energías humanas y recursos financieros que comprometen en la competencia frenética por convertirse en el noticiero más visto, deberían ser canalizados en otra dirección. Un equipo de redactores noche a noche sale a cazar asesinatos, riñas callejeras, accidentes de tránsito, ebrios escandalosos, delincuentes perseguidos por la policía; para luego servirlos en el desayuno como parte de la dieta diaria de los televidentes.

\section{El foso digital}

En relación a la quinta hipótesis de Tuffanelli e Ianes enunciada como el foso digital, desde nuestra perspectiva, situación y realidad de país dependiente, es oportuno señalar y destacar que en nuestro medio se produce una doble "desarticulación" de este foso digital. Primero, el foso que existe al interior de cada uno de nuestros países entre las personas con acceso a las bondades y virtualidades del mundo cibernético y aquellas que no gozan de esta posibilidad. Osencillamente el tema de la conectividad cibernética en el mundo urbano y el mundo rural en los países dependientes. Segundo, el inmenso foso existente entre el vertiginoso desarrollo y uso de las TICs en los países metropolitanos y el incipiente, limitado y precario desarrollo de lo digital en países como los nuestros. Un foso que nos coloca en profunda desventaja económica, política, en fin cultural, frente a los países metropolitanos.

Tufanelli e Ianes a propósito del foso digital colocan los siguientes temas, que de ninguna manera son ajenos a la realidad de nuestros países, cuya peculiaridad consiste en verse sometidos con mayor intensidad a la problemática que ha traído consigo la era digital postmoderna. Estos pedagogos italianos distinguen entre aprendizaje escolar y aprendizaje extra-escolar (digital). Y por supuesto las tensiones a la que se ven sometidas todos los factores humanos y los recursos, que concurren en el proceso enseñanza-aprendizaje al realizarse la cohabitación y eventualmente la transición entre la enseñanza escolar y la extra-escolar.

Asimismo Tuffanelli e Ianes, distinguen muy claramente las diferencias de aptitudes, habilidades y destrezas que existen para aprender y dominar las nuevas tecnologías, entre los nativos de la era digital y los migrantes digitales (nacidos antes de los años 90). Es muy común en el mundo actual, que las personas nacidas en la galaxia gutemberg, o sea antes de los años 90, para aprender a operar algunos mecanismos de aparatos electrónicos debemos consultar a los menores de la casa generalmente nacidos en la era digital.

También los autores de La gestión de la clase señalan que:

Algunos psicólogos de la Real Sociedad de Medicina han demandado al gobierno inglés, vetar o al menos limitar el empleo de computadoras en la escuela por parte de los niños menores de

9 años, sosteniendo, que el uso precoz parece dañar el aprendizaje escolar. Es absolutamente 
necesario que el niño aprenda a descubrir primero el mundo real que el mundo virtual. La misma velocidad de ejecución del PC puede inducir al pequeño a delegar a la máquina aquellas operaciones mentales que necesariamente debe realizar, con el resultado de no aprender a fijar los nexos entre los elementos. Este problema no es privativo de los niños, sino que atañe también a los adultos. De hecho la enorme cantidad de información que absorbemos vía virtual, pone en riesgo de superar la capacidad de elaboración de las neuronas, con el resultado que nuestro cerebro entorpecido permanece indiferente a los problemas que nos circundan.

La doble articulación del foso digital en las sociedades dependientes y países atrasados como los nuestros es un obstáculo a investigar, analizar y superar para realizar una pedagogía de la cultura de paz a nivel global.

\section{La crisis de los adultos}

La sexta hipótesis está enunciada por Tuffanelli e Ianes, como la crisis de los adultos. Por crisis del adulto los autores comprenden la pérdida de autoridad no solo del profesor o la profesora, sino del adulto en general (padres y tutores entre otros). Lo que entra en crisis en los adultos, son los roles específicos del padre y la madre; cada día se ve menos en nuestras sociedades, al padre fijando las reglas y ejercitando la disciplina; la madre siendo el centro afectivo, con un estilo más permisivo, más indulgente, pronta a dar afecto y protección a los hijos.

Padre y madre conforman los elementos de un código materno-paterno dentro de una familia mono nuclear que ha entrado en crisis y ha devenido en familia móvil, en una sociedad postmoderna donde se evidencia la crisis de lo sagrado, la crisis de la política, la misma crisis de la educación y la inserción masiva de la mujer en el mercado laboral, elemento este último determinante en los cambios de patrones. El modelo de familia mono nuclear ha entrado en crisis y ha mutado a otras formas de organización de la familia propias de nuestro tiempo y de su consabida fragmentariedad postmoderna.

Estas nuevas formas de organización de la familia están ocurriendo tanto en los países metropolitanos como en los periféricos e indudablemente tienen que ajustarse todos los anillos de la esfera desde la perspectiva de construcción de una nueva cultura, la cultura de paz. Entre esos anillos que padecen sus propias crisis de transformación particular y de articulación sistemática, encontramos a la familia, la educación, los medios de comunicación social, la sociedad política, el estado, la iglesia, la economía, etcétera. Es decir, los grandes sujetos de la cultura de paz.

\section{BIBLIOGRAFÍA}

Arríen, Juan Bautista: Introducción a la violencia en la educación de Nicaragua en el marco del estudio "Historia y Violencia en Nicaragua", en el volumen colectivo, Historia y Violencia en Nicaragua, editorial Nos-Otros, UPOLI, Managua, 2012.

García, Azucena: Analfabetismo: todavía uno de los males que afectan a la población mundial, Eroski Consumer en Internet.

Lovo, Anastasio: Cultura de Paz - Historia, epistemología y textos-, editorial Nos-Otros UPOLI, Managua, 2012.

Lucio Gil, Rafael: El dolor de ser maestro, nota informativa de El Nuevo Diario, 29 de junio de 2013.

Rothschuh Villanueva, Guillermo: Punto sobre la íes, editorial CINCO, Managua, 2012.

Torres Pérez, Wulfrano: Los niños mejicanos secuestrados por la televisión, en http://www.statuspuebla.com.mx/ index.php?option $=$ com content $\&$ view $=$ article $\&$ id $=2013$

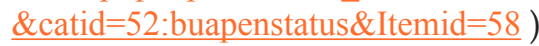

Tuffanelli, Luigi e Ianes, Dario: La gestione Della classe, editorial Ericsson, Trento, 2011. Todas las citas y referencias a esta obra, han sido producto de una traducción libre del italiano al español realizada por Anastasio Lovo.

Tünnermann Bernheim, Carlos: El salario de los maestros, artículo de opinión publicado en El Nuevo Diario, 5 de mayo 2010. 